\title{
Scaling of $\boldsymbol{P}$-wave excitation energies in heavy-quark systems
}

\author{
Marek Karliner, ${ }^{1, *}$ and Jonathan L. Rosner, ${ }^{2, \dagger}$ \\ ${ }^{1}$ School of Physics and Astronomy Raymond and Beverly Sackler Faculty of Exact Sciences \\ Tel Aviv University, Tel Aviv 69978, Israel \\ ${ }^{2}$ Enrico Fermi Institute and Department of Physics University of Chicago, \\ 5640 South Ellis Avenue, Chicago, Illinois 60637, USA
}

(Received 28 August 2018; published 26 October 2018)

\begin{abstract}
A simple regularity in anticipating P-wave excitation energies of states with heavy quarks is noted. It can apply to systems such as the negative-parity $\Sigma_{c}, \Sigma_{b}$, and $\Omega_{c}, \bar{Q} Q$ quarkonia, and the bottom-charmed meson $B_{c}$. When one subtracts a term accounting for phenomenological energies of heavy quarks binding with one another in S-waves, the residual excitation energies display an approximately linear behavior in the reduced mass of constituents, all the way from the $\Lambda$ to the $\Upsilon$.
\end{abstract}

DOI: 10.1103/PhysRevD.98.074026

\section{INTRODUCTION}

The LHCb experiment, working at the CERN Large Hadron Collider, has observed a number of new baryons containing heavy quarks, including a series of five excited $\Omega_{c}=c s s$ resonances [1] and a new $\Xi_{b}^{-}=b s d$ resonance [2]. These have been interpreted, though not uniquely, as, respectively, P-wave excitations of the ground state $\Omega_{c}$ [3-8] and one or more P-wave excitations of the ground state $\Xi_{b}^{-}$ [9]. We seek simple methods for confirming these assignments. Furthermore, it has been of interest to estimate the Pwave excitation energies for $\Sigma_{c}$ and $\Sigma_{b}$ states [10] as well as for the $B_{c}=b \bar{c}$ system (see, e.g., [11-15]).

Spurred by these developments, we asked whether there is a simple way of estimating P-wave excitation energies without the use of the two-body or three-body Schrödinger equation, its relativistic analogue, or other methods such as lattice quantum chromodynamics. To our surprise, there appears to be an approximate method which, while not perfect, probably suffices as a guideline to whether a given state is a $\mathrm{P}$-wave candidate.

The method builds upon a constituent-quark treatment which was used to predict successfully [16] the mass of the $\Xi_{c c}^{++}=c c u$ baryon subsequently discovered by LHCb [17]. Account was taken of quark masses, hyperfine interactions, and $\mathrm{S}$-wave binding terms $B\left(q_{1} q_{2}\right)$ involving any quark pairs where one quark is heavier than $u, d$ and the

\footnotetext{
*marek@proton.tau.ac.il

rosner@hep.uchicago.edu
}

Published by the American Physical Society under the terms of the Creative Commons Attribution 4.0 International license. Further distribution of this work must maintain attribution to the author(s) and the published article's title, journal citation, and DOI. Funded by SCOAP . other heavier than $s$. These binding terms are obtained phenomenologically by comparing masses of hadrons containing a single heavy quark (e.g., $q_{1}$ or $q_{2}$ ) with ones containing two heavy quarks (e.g., $q_{1} \bar{q}_{2}$ ). We find that when these binding terms are taken into account in calculating S-P mass differences, the residual energy differences $\Delta E_{R}$ depend approximately linearly on the reduced mass $\mu_{12}=m_{1} m_{2} /\left(m_{1}+m_{2}\right)$ of the pair. This behavior extends from the $\Lambda=u d s$ baryon all the way up to the $\Upsilon(1 S)$ and their respective P-wave excitations.

We lay out the tools for our estimates in Sec. II, describing assumed quark masses and binding terms. The ground rules for quoting S-P splittings are also given. We quote the observed S-P splittings for a number of pairs in Sec. III. The effects of binding terms, if any, are considered in Sec. IV, giving rise to residual energy differences $\Delta E_{R}$ which are plotted as functions of reduced mass. An approximately linear dependence is seen. In baryonic cases the problem is reduced to a two-body one by assuming one quark is excited with respect to two others which remain in a relative $S$-wave.

The linear dependence of $\Delta E$ on reduced mass is used in Sec. V to predict several quantities which were only crudely estimated before. These include P-wave excitation energies for $\Sigma_{c}$ and $\Sigma_{b}$ states [10] and for $\Omega_{c}$ states [3]. Predictions for $\Xi_{b}$ and $B_{c}$ are also given and compared with others in the literature. Section VI is devoted to a discussion of the possible source of the observed regularity, and a brief conclusion.

\section{TOOLS}

We use separate constituent-quark masses for mesons and baryons [16]. They are summarized in Table I. Analysis of $\mathrm{S}$-wave mesons and baryons makes use of binding terms $B\left(q_{1} q_{2}\right)$, also from Ref. [16], summarized in Table II. 
TABLE I. Quark masses in MeV used in this analysis.

\begin{tabular}{lcc}
\hline \hline Quark & In a meson & In a baryon \\
\hline$u, d$ & $m_{u, d}^{m}=310$ & $m_{u, d}^{b}=363$ \\
$s$ & $m_{s}^{m}=483$ & $m_{s}^{b}=538$ \\
$c$ & $m_{c}^{m}=1663.3$ & $m_{c}^{b}=1710.5$ \\
$b$ & $m_{b}^{m}=5003.8$ & $m_{b}^{b}=5043.5$ \\
\hline \hline
\end{tabular}

TABLE II. Pair binding terms $B\left(q_{1} q_{2}\right)$ in $\mathrm{MeV}$ used in this analysis.

\begin{tabular}{lcc}
\hline \hline Pair $q_{1} q_{2}$ & $B\left(q_{1} q_{2}\right)$ & $B\left(q_{1} \bar{q}_{2}\right)$ \\
\hline$c s$ & 35.0 & 70.0 \\
$b s$ & 41.8 & 83.6 \\
$c c$ & 129 & 258 \\
$b c$ & 170.8 & 341.5 \\
$b b$ & 281.4 & 562.8 \\
\hline \hline
\end{tabular}

These terms were calculated by comparing the masses of spin-averaged S-wave bound states (e.g., for charmonium) with the sum of their constituent-quark masses as determined from hadrons containing a single heavy quark (e.g., $\Lambda_{c}$ ).

\section{S-P SPLITTINGS}

\section{A. Baryons}

Unless otherwise specified, we take all masses from the 2018 Particle Data Group listings [18]. We consider

TABLE III. Masses of ground state baryons and their orbital excitations $\Delta E_{P-S}$, in $\mathrm{MeV}$. Here $\Delta E_{P-S}$ denotes the difference between spin-weighted average $\mathrm{P}$-wave and $\mathrm{S}$-wave masses.

\begin{tabular}{llllll}
\hline \hline State & \multicolumn{1}{c}{$1 / 2^{+}$} & $1 / 2^{-}$ & \multicolumn{1}{c}{$3 / 2^{-}$} & $\bar{M}_{P}$ & $\Delta E_{P-S}$ \\
\hline$\Lambda$ & 1115.683 & 1405.1 & 1519.5 & 1481.37 & 365.68 \\
$\Lambda_{c}$ & 2286.46 & 2592.25 & 2628.11 & 2616.16 & 329.70 \\
$\Lambda_{b}$ & 5619.60 & 5912.20 & 5919.92 & 5917.35 & 297.75 \\
$\Xi_{c}$ & $2469.37^{\mathrm{a}}$ & $2792.2^{\mathrm{a}}$ & $2818.4^{\mathrm{a}}$ & 2809.6 & 340.3 \\
$\Omega_{c}{ }^{\mathrm{b}}$ & 2742.33 & \multicolumn{2}{c}{ See note $^{\mathrm{c}}$} & 3079.94 & 337.61 \\
\hline \hline
\end{tabular}

${ }^{\mathrm{a}}$ Error-weighted isospin average.

${ }^{\mathrm{b}}$ Spin-averages of ground state and assumed P-wave states from Ref. [3].

${ }^{\mathrm{c}}(2,2,1)$ states with $J=(1 / 2,3 / 2,5 / 2)$, cf. Ref. [3]. baryons with excitation of a spinless (scalar) diquark except in the case of $\Omega_{c}=c s s$, where we consider the spin-1 ss diquark to be excited by one unit of orbital angular momentum with respect to the charmed quark [3].

We take the masses listed in Table III to calculate the spin-averaged S-P splittings shown. The masses of excited states are calculated using averages $\bar{M}_{P}$ weighted by $2 J+1$ factors, where $J$ is the spin of the resonance. Small uncertainties in masses are not quoted.

\section{B. Mesons}

We consider only those systems for which the spinaveraged ground state and P-wave masses can be calculated. They are $c \bar{s}$ (" $D_{s}$ "), $c \bar{c}$, and $b \bar{b}$. For $c(\bar{u}, \bar{d})$ not all candidates for the 1P level are firmly established, while for $b(\bar{u}, \bar{d})$ a spin-zero meson and one of two predicted spin-1 mesons are still missing (see Sec. V B). For $b \bar{c}$ (" $B_{c}$ ") no P-wave states have been seen, but their masses have been predicted (see Sec. V F). The relevant masses are shown in Table IV. Spin averaged masses are

$\bar{M}_{S} \equiv\left[M\left({ }^{1} S_{0}\right)+3 M\left({ }^{3} S_{1}\right)\right] / 4$,

$\bar{M}_{P} \equiv\left[M\left({ }^{3} P_{0}\right)+3 M\left({ }^{3} P_{1}\right)+3 M\left({ }^{1} P_{1}\right)+5 M\left({ }^{3} P_{2}\right)\right] / 12$.

\section{RESIDUAL ENERGY DIFFERENCES $\Delta E_{R}$}

We now calculate residual energy differences $\Delta E_{R} \equiv$ $\Delta E_{P-S}-\sum B$ for the above systems, where $\sum B$ denotes the sum of $B\left(q_{1} q_{2}\right)$ over all relevant heavy quarks $q_{1}$ and $q_{2}$ (cf. Table II). The results are shown in Table V. Here $\left[q_{1} q_{2}\right]$ denotes a spinless color-antitriplet diquark, while $(s s)$ denotes a spin-1 color-antitriplet diquark. We quote isospin-averaged masses where appropriate, letting $q$ stand for $u$ or $d$.

Whereas the quantities $\Delta E_{P-S}$ are not monotonic functions of the reduced mass $\mu_{12}$, when the binding energies $B$ are subtracted from them, the residual energies $\Delta E_{R}$ are crudely arranged along a straight line, as shown in Fig. 1. A linear fit to the eight experimentally known values in Table V gives the result

$$
\Delta E_{R}=\left(417.37-0.2141 \mu_{12}\right) \mathrm{MeV} .
$$

TABLE IV. Masses of ground state mesons and their orbital excitations, in MeV.

\begin{tabular}{llllllllll}
\hline \hline State & $M\left({ }^{1} S_{0}\right)$ & $M\left({ }^{3} S_{1}\right)$ & $\bar{M}_{S}{ }^{\mathrm{a}}$ & $M\left({ }^{3} P_{0}\right)$ & $M\left({ }^{3} P_{1}\right)$ & $M\left({ }^{1} P_{1}\right)$ & $M\left({ }^{3} P_{2}\right)$ & $\bar{M}_{P}{ }^{\mathrm{b}}$ & $\Delta E_{P-S}$ \\
\hline$D_{s}$ & 1968.34 & 2112.2 & 2076.2 & 2317.7 & $2459.5^{\mathrm{c}}$ & $2535.1^{\mathrm{c}}$ & 2569.1 & 2512.3 & 436.0 \\
$c \bar{c}$ & 2983.4 & 3096.9 & 3068.5 & 3414.71 & 3510.67 & 3525.38 & 3556.17 & 3525.3 & 456.8 \\
$b \bar{b}$ & 9399.0 & 9460.3 & 9445.0 & 9859.44 & 9892.78 & 9899.73 & 9912.21 & 9899.7 & 454.8 \\
\hline \hline
\end{tabular}

${ }^{\mathrm{a}}$ Spin-averaged ground state mass.

${ }^{\mathrm{b}}$ Spin-averaged P-wave mass.

${ }^{\mathrm{c}}$ Orthogonal mixtures of ${ }^{3} P_{1}$ and ${ }^{1} P_{1}$ states. 
TABLE V. Residual energy differences $\Delta E_{R}$ and corresponding reduced masses, in $\mathrm{MeV}$.

\begin{tabular}{lcccccccc}
\hline \hline System & $q_{1}$ & $q_{2}$ & $m_{1}$ & $m_{2}$ & $\mu_{12}$ & $\Delta E_{P-S}$ & $\sum B$ & $\Delta E_{R}$ \\
\hline$\Lambda$ & {$[u d]$} & $s$ & 576.0 & 538 & 278.2 & 365.7 & 0 & 365.7 \\
$\Lambda_{c}$ & {$[u d]$} & $c$ & 576.0 & 1710.5 & 430.9 & 329.7 & 0 & 329.7 \\
$\Lambda_{b}$ & {$[u d]$} & $b$ & 576.0 & 5043.5 & 517.0 & 297.8 & 0 & 297.8 \\
$\Xi_{c}$ & {$[q s]$} & $c$ & 799.8 & 1710.5 & 545.0 & 340.3 & 35.0 & 305.3 \\
$\Omega_{c}$ & $(s s)$ & $c$ & 1098.8 & 1710.5 & 669.0 & 337.6 & 70.0 & 267.6 \\
$D_{s}$ & $c$ & $s$ & 1663.3 & 483 & 374.3 & 436.0 & 70.0 & 366.0 \\
$c \bar{c}$ & $c$ & $c$ & 1663.3 & 1663.3 & 831.6 & 456.8 & 258.0 & 198.8 \\
$b \bar{b}$ & $b$ & $b$ & 5003.8 & 5003.8 & 2501.9 & 454.8 & 563 & -108.2 \\
\hline \hline
\end{tabular}

The root-mean-square deviation of the data from this fit is 18.7 MeV. We discuss some consequences of this regularity, if it is to be taken seriously, in the next section.

\section{CONSEQUENCES AND PREDICTIONS}

\section{A. $\Sigma_{c}$ and $\Sigma_{b}$ baryons}

In Ref. [10] a linear extrapolation of excitation energy was used to estimate the S-P wave splittings for $\Sigma_{c}$ and $\Sigma_{b}$ baryons. The present discussion gives support to that assumption. The parameters of the present linear fit give slightly different values of $\Delta E_{R}$, as shown in Table VI. For the states in this table, there are no $B$ terms, so $\Delta E_{P-S}=\Delta E_{R}$.

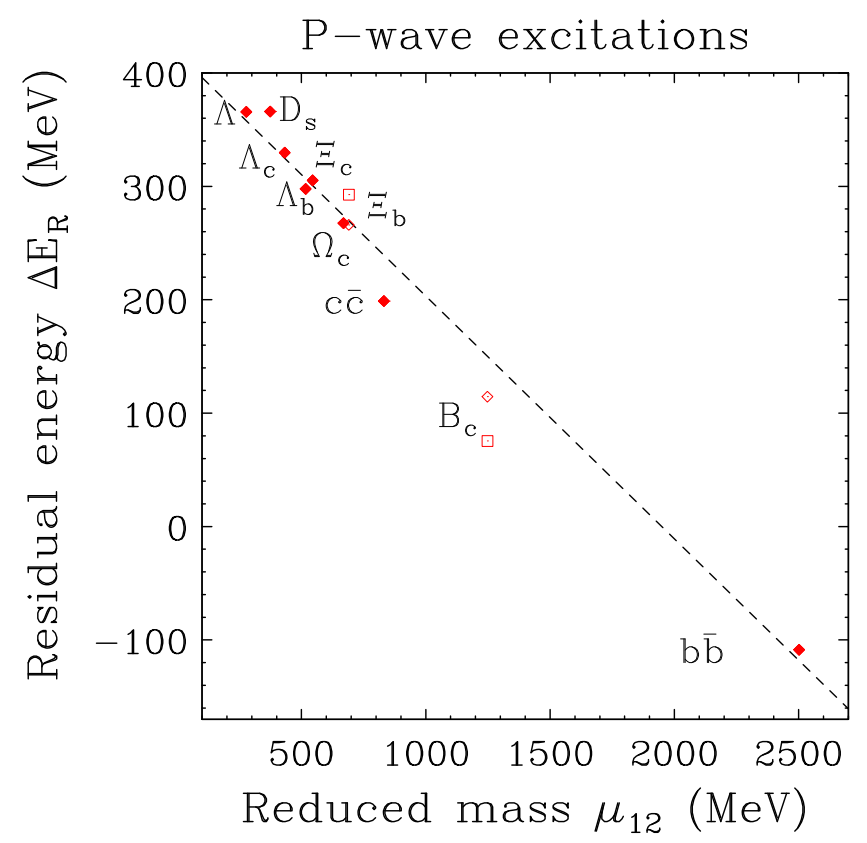

FIG. 1. Residual energies $\Delta E_{R}$ as functions of reduced mass $\mu_{12}$. Dashed line: fit of Eq. (2). Filled diamonds denote data used in the fit. Theoretical predictions for $\Xi_{b}\left(\right.$ Sec. V E) and $B_{c}$ (Sec. V F) systems are plotted as hollow diamonds and hollow squares.
TABLE VI. Values of $\Delta E_{R}=\Delta E_{P-S}$ predicted by linear fit of Eq. (2) compared with those of Ref. [10].

\begin{tabular}{lcccccccc}
\hline \hline & & & & & & \multicolumn{2}{c}{$\Delta E_{R}$} \\
\cline { 5 - 9 } & & & $m_{1}$ & $m_{2}$ & $\mu_{12}$ & & \\
State & $q_{1}$ & $q_{2}$ & $(\mathrm{MeV})$ & $(\mathrm{MeV})$ & $(\mathrm{MeV})$ & Ref. [10] & Eq. (2) \\
\hline$\Sigma$ & $(u u)$ & $s$ & 776 & 538 & 317.7 & 357.5 & 349.3 \\
$\Sigma_{c}$ & $(u u)$ & $c$ & 776 & 1710.5 & 533.8 & 290.9 & 303.1 \\
$\Sigma_{b}$ & $(u u)$ & $b$ & 776 & 5043.5 & 672.5 & 238.8 & 273.4 \\
\hline \hline
\end{tabular}

\section{B. Charm and bottom mesons}

The reduced masses for $D$ and $B$ mesons are displayed in Table VII. They lead to predictions via Eq. (2) of $\Delta E_{R}$, which is equal to $\Delta E_{P-S}$ because the binding correction $B$ is zero.

In order to compare these predictions with experiment, one must know the masses of all four P-wave states. Our partial information is summarized in Table VIII. The $D$ and $B$ mass eigenstates have $j$ (the vector sum of light-quark spin and orbital angular momentum) equal to $1 / 2$ or $3 / 2$. Those with $j=3 / 2$ (total $J=1,2$ ) decay predominantly via $\mathrm{D}$ waves, are narrow, and are firmly established [18]. Those with $j=1 / 2(J=0,1)$ are expected to decay via $\mathrm{S}$ waves and are very broad, with consequent mass uncertainty. The $j=1 / 2 D$ mesons would satisfy the linear fit if their widths, exceeding $200 \mathrm{MeV}$, were included as error bars. No candidates for the $j=1 / 2 B$ states have been identified. They would have to be considerably lighter than the $j=3 / 2$ states if they were to obey the prediction in Table VII. The outlier nature of $D$ and $B$ states is further discussed in Sec. VI.

The predicted spin-averaged P-wave mass for $B_{s}$ is low enough that the $j=1 / 2 B_{s} \mathrm{P}$-wave states are probably below the respective $B K$ and $B^{*} K$ thresholds for the $J=0$ and $J=1$ states. Thus, like the $D_{s 0}(2317)$ and $D_{s 1}(2460)$ (see below), they are expected to be very narrow, decaying only via $B_{s 0} \rightarrow \gamma B_{s}^{*}$ and $B_{s 1} \rightarrow \gamma B_{s}$ or $\gamma B_{s}^{*}$, or with isospinviolating processes involving $\pi^{0}$ emission. The properties of these states have been discussed in Refs. $[19,20]$.

\section{C. $D_{s}$ mesons}

The observed masses of $D_{s 0}(2317)$ and $D_{s 1}(2460)$ were considerably below predictions of potential models, leading to some initial surprise. The present regularity (Fig. 1)

TABLE VII. Calculation of $\Delta E_{R}$ and $\Delta E_{P-S}=\Delta E_{R}+$ $B\left(q_{1} q_{2}\right)$ for $D, B$, and $B_{s}$ mesons, based on linear fit of Eq. (2). Masses in MeV.

\begin{tabular}{lcccccccc}
\hline \hline State & $q_{1}$ & $q_{2}$ & $m_{1}$ & $m_{2}$ & $\mu_{12}$ & $\Delta E_{R}$ & $B\left(q_{1} q_{2}\right)$ & $\Delta E_{P-S}$ \\
\hline$D$ & $c$ & $q$ & 1663.3 & 310 & 261.3 & 361.4 & 0 & 361.4 \\
$B$ & $b$ & $q$ & 5003.8 & 310 & 291.9 & 354.9 & 0 & 354.9 \\
$B_{s}$ & $b$ & $s$ & 5003.8 & 483 & 440.5 & 323.1 & 83.6 & 406.7 \\
\hline \hline
\end{tabular}


TABLE VIII. Masses for calculating S-P splitting in charmed and bottom mesons. Error-weighted averages over charge states unless otherwise indicated.

\begin{tabular}{llllcllll}
\hline \hline State & $M\left({ }^{1} S_{0}\right)$ & $M\left({ }^{3} S_{1}\right)$ & $\bar{M}_{S}{ }^{\mathrm{a}}$ & $\bar{M}_{P}{ }^{\mathrm{b}}$ (predictions) & $M\left({ }^{3} P_{0}\right)$ & $j=1 / 2$ & $j=3 / 2$ & $M\left({ }^{3} P_{2}\right)$ \\
\hline$D$ & 1867.24 & 2008.56 & $1973.23^{\mathrm{a}}$ & 2334.6 & $2349.2^{\mathrm{c}}$ & Note $^{\mathrm{d}}$ & 2420.9 & 2461.1 \\
$B$ & 5279.48 & 5324.65 & $5313.36^{\mathrm{a}}$ & 5668.2 & $\cdots$ & $\cdots$ & 5726.0 & 5738.4 \\
$B_{s}$ & 5366.89 & 5415.4 & $5403.3^{\mathrm{a}}$ & 5810.0 & $\cdots$ & $\cdots$ & 5828.63 & 5839.85 \\
\hline \hline
\end{tabular}

${ }^{\mathrm{a}}$ Spin-averaged ground state mass.

${ }^{\mathrm{b}}$ Spin-averaged P-wave mass predicted from Eq. (2).

${ }^{c}$ Error-weighted isospin average width $235.7 \mathrm{MeV}$.

${ }^{\mathrm{d}}$ Neutral candidate: $M=2427 \pm 40 \mathrm{MeV}, \Gamma=384_{-110}^{+130} \mathrm{MeV}$.

indeed supports the picture of these states as lying below those predictions.

\section{D. $\boldsymbol{\Omega}_{c}$ baryons}

The residual energy $\Delta E_{R}$ for the five narrow $\Omega_{c}$ states observed by LHCb [1] lies right on the linear fit, supporting their assignment as five P-wave states [3-8] and disfavoring an alternate assignment (see, e.g., [3]) in which the two highest states are $2 \mathrm{~S}$ excitations and two lower-mass $\mathrm{P}$-wave states remain to be discovered.

\section{E. $\Xi_{b}$ baryons}

In Table IX we compare a recent prediction [9] for the masses of P-wave excitations of the scalar $[s q]$ quark in $\Xi_{b}$ baryons (Fig. 1, hollow diamond), with an earlier one ([21], Fig. 1, hollow square), and with the result of the linear fit for reduced mass $\mu_{b c}=690.3 \mathrm{MeV}$. The fit is more consistent with the later prediction.

\section{F. $B_{c}$ states}

One can obtain a value of $\Delta E_{P-S}$ for the $B_{c}$ system by interpolating between the nearly equal values for the $c \bar{c}$ and $b \bar{b}$ systems, as one might expect if the interquark potential is close to the logarithmic one proposed in Ref. [22]. One thus obtains $\Delta E_{P-S}=456 \mathrm{MeV}$, corresponding to the open diamond in Fig. 1 when a binding term of $341.5 \mathrm{MeV}$ is taken into account. An early potential-model prediction [11] was $\Delta E_{P-S}=417 \mathrm{MeV}$, corresponding to the open square in Fig. 1. Subsequent calculations of $\Delta E_{P-S}$ gave

TABLE IX. Values of $\Delta E_{P-S}$ and $\Delta E_{R}$ for $\Xi_{b}$ states from models compared with predictions of linear fit (2). Masses in $\mathrm{MeV}$.

\begin{tabular}{|c|c|c|c|c|c|}
\hline $1 / 2^{+}$ & $1 / 2^{-}$ & $3 / 2^{-}$ & $\bar{M}_{P}$ & $\Delta E_{P-S}$ & $\Delta E_{R}$ \\
\hline \multirow[t]{3}{*}{$5792.19^{\mathrm{a}}$} & $6096^{\mathrm{b}}$ & $6102^{\mathrm{b}}$ & 6100 & 307.8 & 266.0 \\
\hline & $6120^{c}$ & $6230^{\mathrm{c}}$ & 6126.7 & 334.5 & 292.7 \\
\hline & \multicolumn{3}{|c|}{ Calculated from Eq. (2) } & 311.4 & 269.6 \\
\hline
\end{tabular}

\footnotetext{
${ }^{\mathrm{a}} \mathrm{Spin}$-averaged ground state mass.

${ }^{\mathrm{b}}$ Ref. [9].

${ }^{\mathrm{c}}$ Ref. [21].
}

430, 427, and $427 \mathrm{MeV}$ in Refs. [12-14], respectively. The prediction of Eq. (2), using a reduced mass of $m_{c} m_{b} /\left(m_{c}+m_{b}\right)=1248.3 \mathrm{MeV}$, is $\Delta E_{R}=150.1 \mathrm{MeV}$, or $\Delta E_{P-S}=491.6 \mathrm{MeV}$, considerably larger than any of the above values.

\section{DISCUSSION AND CONCLUSIONS}

The binding terms $B$ used to calculate $\Delta E_{R}$ represent corrections to the picture of spectra due to constituentquark masses and hyperfine terms [23], when quarks are heavy enough to experience the short-distance Coulomblike force of single gluon exchange. In a purely Coulombic potential $V(r)=-(4 / 3) \alpha_{s} / r$ the energy levels are given by $E_{n}=-\left[(4 / 3) \alpha_{s}\right]^{2} \mu /\left(2 n^{2}\right)$. In the simplest approximation the $\mathrm{P}$-wave excitation energy is given by $\Delta E_{P-S}=E_{2}-E_{1}$. We have subtracted the S-wave binding energy $B$ from this P-wave excitation energy to obtain the residual energy difference

$$
\Delta E_{R}=\Delta E_{P-S}-B
$$

In our convention this S-wave binding energy is positive. In this simple example it is just the minus the ground state energy, $-E_{1}$. The upshot is that here the residual excitation energy is just the energy eigenvalue of the P-wave,

$\Delta E_{R}=E_{2}-E_{1}-B=E_{2}-E_{1}+E_{1}=E_{2}=-\left[(4 / 3) \alpha_{s}\right]^{2} \mu / 8$.

So in this case the slope in Fig. 1 is just $-\left[(4 / 3) \alpha_{s}\right]^{2} \mu / 8$.

In a more realistic potential with a confining piece the slope will be different and there is likely to be also a constant term. For light quarks $(u, d, s)$ the use of constituent-quark masses means that it is not necessary to subtract a $B$ term; the constituent-quark masses already embody such a term. Nonetheless, the negative slope in the relation between residual energy and reduced mass is generic. It just reflects the fact that the P-wave energy (as opposed to energy splitting) is negative.

This is surprising, as relativistic corrections (important even for systems as heavy as bottomonium) do not depend 
purely on the reduced mass. This is true for quantum electrodynamics, as shown by the comparison between positronium and the hydrogen atom [24]. The linear dependence of residual energy must be the result of compensating effects, not some fundamental relation. What we have done is to construct a phenomenological "bridge" between confinement and short-distance Coulomb-like behavior. This picture then explains why the $B$ and $D$ mesons are outliers. Their radii are of order $1 / \Lambda_{\mathrm{QCD}}$, rather than $1 /\left(\alpha_{s} \mu\right)$. The fact that $\alpha_{s}$ runs between $\mu=500 \mathrm{MeV}$ and $2500 \mathrm{MeV}$ will make the slope slightly scale dependent.
The potential for learning about P-wave excitations of heavy-quark baryons and mesons makes the present discussion timely. Consequences have been noted for charmed and bottom-flavored baryons and mesons. It will be interesting to see if some of these regularities are further supported by experiment.

\section{ACKNOWLEDGMENTS}

We thank Sheldon Stone and Tomasz Skwarnicki for awakening our interest in P-wave excitations of heavyquark systems and for helpful comments.
[1] R. Aaij et al. (LHCb Collaboration), Phys. Rev. Lett. 118, 182001 (2017).

[2] R. Aaij et al. (LHCb Collaboration), Phys. Rev. Lett. 121, 072002 (2018).

[3] M. Karliner and J. L. Rosner, Phys. Rev. D 95, 114012 (2017).

[4] M. Padmanath and N. Mathur, Phys. Rev. Lett. 119, 042001 (2017).

[5] W. Wang and R.-L. Zhu, Phys. Rev. D 96, 014024 (2017).

[6] Z. G. Wang, Eur. Phys. J. C 77, 325 (2017).

[7] B. Chen and X. Liu, Phys. Rev. D 96, 094015 (2017).

[8] T. M. Aliev, S. Bilmis, and M. Savci, arXiv:1704.03439.

[9] B. Chen, K. W. Wei, X. Liu, and A. Zhang, Phys. Rev. D 98 , 031502 (2018).

[10] M. Karliner and J. L. Rosner, Phys. Rev. D 92, 074026 (2015).

[11] E. J. Eichten and C. Quigg, Phys. Rev. D 49, 5845 (1994).

[12] D. Ebert, R. N. Faustov, and V. O. Galkin, Phys. Rev. D 67, 014027 (2003).

[13] V. V. Kiselev, A. K. Likhoded, and A. V. Tkabladze, Phys. Rev. D 51, 3613 (1995).
[14] L. P. Fulcher, Phys. Rev. D 60, 074006 (1999).

[15] N. Mathur, M. Padmanath, and S. Mondal, arXiv:1806 .04151 .

[16] M. Karliner and J. L. Rosner, Phys. Rev. D 90, 094007 (2014).

[17] R. Aaij et al. (LHCb Collaboration), Phys. Rev. Lett. 119, 112001 (2017).

[18] M. Tanabashi et al. (Particle Data Group), Phys. Rev. D 98, 030001 (2018).

[19] W. A. Bardeen, E. J. Eichten, and C. T. Hill, Phys. Rev. D 68, 054024 (2003).

[20] M. A. Nowak, M. Rho, and I. Zahed, Acta Phys. Pol. B 35, 2377 (2004) [arXiv:hep-ph/0307102].

[21] D. Ebert, R. N. Faustov, and V. O. Galkin, Phys. Rev. D 84, 014025 (2011).

[22] C. Quigg and J. L. Rosner, Phys. Lett. 71B, 153 (1977).

[23] A. De Rújula, H. Georgi, and S. L. Glashow, Phys. Rev. D 12, 147 (1975).

[24] T. Fulton and P. C. Martin, Phys. Rev. 95, 811 (1954). 